\title{
Mini-implant supported Orthodontic Molar Intrusion: An Unconventional Approach for Pre-implant Prosthetics
}

\author{
${ }^{1}$ Prathmesh R Mehta, ${ }^{2}$ Rishi A Bhimani, ${ }^{3}$ Anand N Badavannavar
}

\begin{abstract}
Aim: To demonstrate two cases in which mini-implant-supported orthodontic molar intrusion has been performed to regain ideal crown height space (CHS) for implant prosthesis.

Background: Tooth loss is associated with esthetic, functional, and psychological consequences on an individual. Replacement of missing teeth by dental implants has gained significant importance due to promising advantages of implants over conventional prosthesis. Edentulism often results in supra-eruption of antagonistic teeth into the edentulous site, resulting in reduced vertical dimension for tooth replacement, posing a restorative challenge for clinicians. Hence, it is necessary to regain the lost vertical dimension prior to the implant prosthesis, either conventionally by endodontic and prosthetic intervention or by orthodontic intrusion with the aid of temporary anchorage devices (TADs). Mini-implants are a category of TADs that provide a minimally invasive technique for molar intrusion.
\end{abstract}

Case report: Two patients with missing mandibular molars desiring tooth replacement presented with supra-eruption of antagonist teeth, posing a difficulty during prosthetic treatment. Orthodontic intrusion using mini-implants was performed to regain the lost vertical space simultaneously along with dental implant placement followed by prosthetic rehabilitation.

Conclusion: The present twin case report demonstrates the successful role of mini-implants in orthodontic molar intrusion for establishing an ideal $\mathrm{CHS}$ for implant prosthesis to reestablish masticatory function.

Clinical significance: The twin case report demonstrates miniimplant-supported orthodontic molar intrusion as a replacement for conventional gross tooth reduction, for regaining the lost vertical space in cases of supra-eruption of maxillary molar associated with edentulism.

Keywords: Implants, Intrusion, Mini-implant, Preprosthetic orthodontics, Temporary anchorage device.

How to cite this article: MehtaPR, Bhimani RA, BadavannavarAN. Mini-implant supported Orthodontic Molar Intrusion: An

\footnotetext{
${ }^{1,2}$ Postgraduate Student, ${ }^{3}$ Reader

${ }^{1,3}$ Department of Orthodontics \& Dentofacial Orthopedics KLE Academy of Higher Education and Research, KLE University's; KLE Vishwanath Katti Institute of Dental Sciences Belagavi, Karnataka, India

${ }^{2}$ Department of Periodontics, KLE Academy of Higher Education and Research, KLE University's; KLE Vishwanath Katti Institute of Dental Sciences, Belagavi, Karnataka, India

Corresponding Author: Rishi A Bhimani, Postgraduate Student, Department of Periodontics, KLE Academy of Higher Education and Research, KLE University's; KLE Vishwanath Katti Institute of Dental Sciences, Belagavi, Karnataka, India Phone: +919833947368, e-mail: rishibhimani@gmail.com
}

Unconventional Approach for Pre-implant Prosthetics. Int J Oral Implantol Clin Res 2017;8(1):26-30.

\section{Source of support: Nil}

Conflict of interest: None

\section{BACKGROUND}

Tooth loss is associated with esthetic, functional, and psychological consequences on an individual. Replacement of missing teeth by dental implants has gained significant importance due to promising advantages of implants over conventional prosthesis.

Edentulism often results in supra-eruption of antagonistic teeth into the edentulous site resulting in reduced vertical dimension for tooth replacement, presenting a restorative challenge for the clinician.

Thus, it necessitates regaining the lost vertical dimension prior to the implant placement, either conventionally by endodontic and prosthetic intervention or by orthodontic intrusion with the aid of TADs.

A TAD is a device, that is temporarily fixed to bone for the purpose of enhancing orthodontic anchorage either by supporting the teeth of the reactive unit or by obviating the need for the reactive unit altogether, which is subsequently removed after use. ${ }^{1}$

The TADs used for molar intrusion are mini-implants or zygomatic plates that aid in achieving ideal results maintaining functional occlusion. Mini-implants can be used in anterior as well as posterior regions to achieve ideal orthodontic results. ${ }^{2}$ Mini-implant-supported intrusion helps to regain the lost vertical dimension for the implant prosthesis.

Thus, the purpose of this article is to demonstrate two cases in which molar intrusion was achieved using orthodontic mini-implants to regain ideal CHS for implant prosthesis.

\section{CASE REPORTS}

\section{Case 1}

A 27-year-old male patient reported with a chief complaint of missing lower left first molar (36) and desired for replacement of the same. The tooth was extracted 6 months earlier due to failed endodontic therapy. On clinical examination, the patient presented with ideal overjet and overbite and a class I molar relationship on the right side and mutilated 


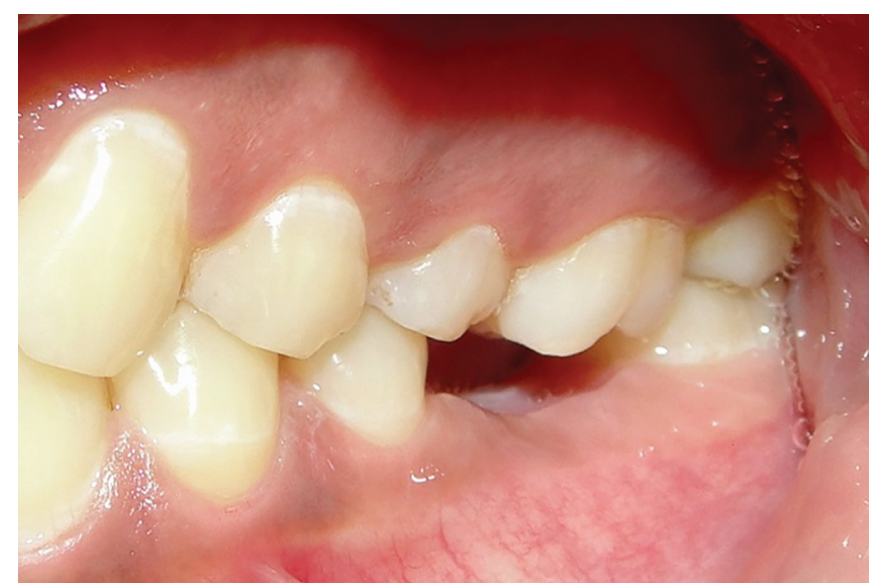

Fig. 1: Pretreatment clinical photograph showing supra-eruption of 26 and reduced $\mathrm{CHS}$ for prosthesis placement

occlusion on the left side. Upper left first molar (26) was supra-erupted reducing the space between the mandibular ridge crest and buccal cusp of 26 to $3.58 \mathrm{~mm}$ (Fig. 1). For the replacement of 36 , two treatment modalities were advised, either dental implant or fixed partial denture prosthesis. The patient opted for an implant prosthesis.
Diagnostic casts were prepared and a facebow record was made, and the patient was guided in centric relation by bilateral manipulation and the bite record was made using bite registration silicone. The vertical space was measured after mounting the models in centric relation on a semiadjustable articulator using a digital Vernier caliper (Mitutoyo Absolut, Mitutoyo Corporation, Japan) (Fig. 2). The treatment plan included placement of one implant in 36, with simultaneous intrusion of 26 using orthodontic mini-implants.

\section{Implant Placement in the Mandible}

Radiographic examination and bone sounding using a bone caliper were done in the region of implant placement. Adequate bone height and width along with soft tissue thickness were present. A surgical stent was prepared to guide the pilot drill into the alveolar crest (Fig. 3). A conventional mucoperiosteal flap was reflected and sequential osteotomies with ascending drill diameters were performed. A $5 \times 10 \mathrm{~mm}$ implant (Noris Medical, Israel) was placed and the flap was sutured (Fig. 4). The

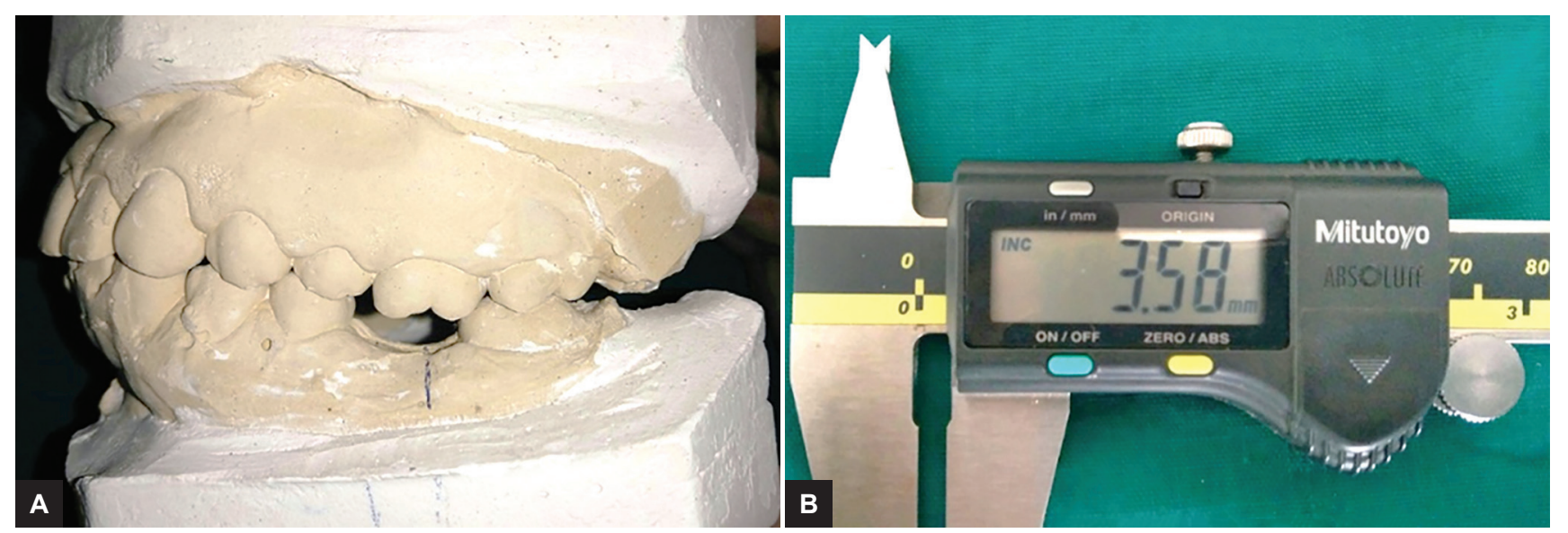

Figs 2A and B: Pretreatment cast showing CHS reduced to $3.58 \mathrm{~mm}$

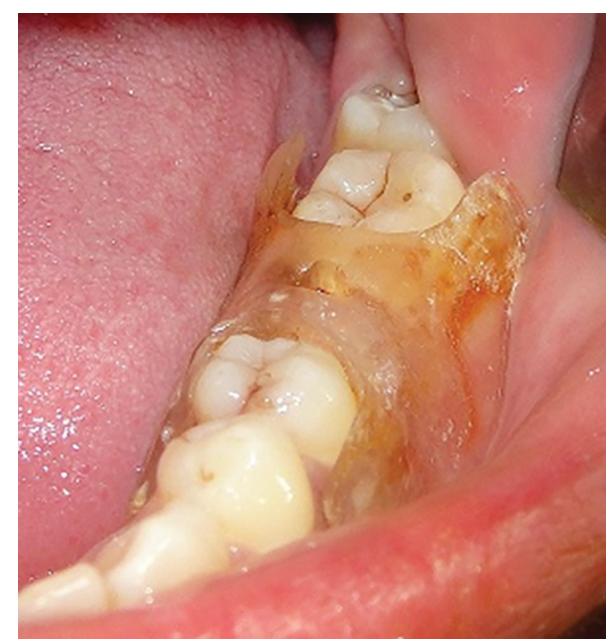

Fig. 3: Surgical stent for implant placement

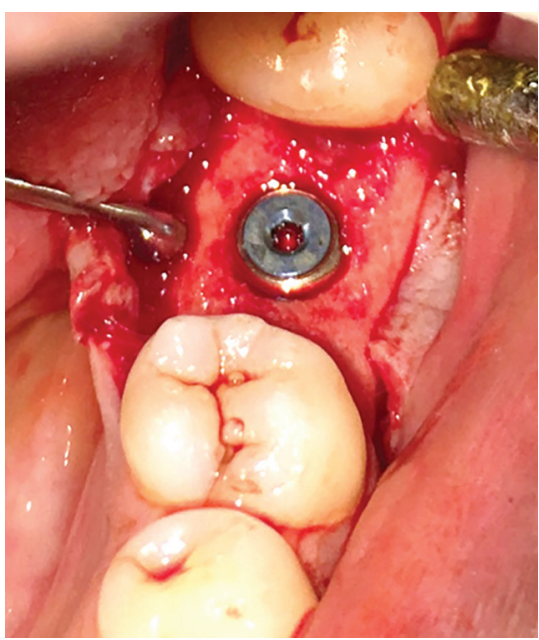

Fig. 4: Implant placed in the edentulous site 


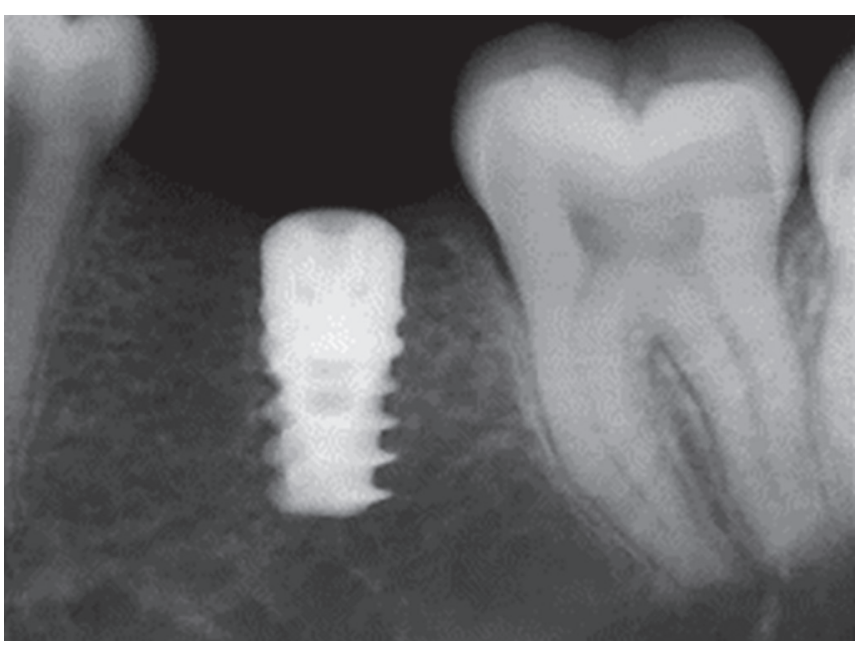

Fig. 5: Radiovisuograph with dental implant

implant was allowed to osseointegrate for 3 months (delayed loading protocol) $^{3}$ (Fig. 5).

\section{Mini-implant Placement in Maxilla}

1 month prior to implant placement in the mandible, mini-implants were placed in the maxillary arch. On the buccal side, a $2 \times 9 \mathrm{~mm}$ mini-implant (S.K. Surgicals) was placed between 25 and 26 and palatally, a $2 \times 6 \mathrm{~mm}$ (S.K. Surgicals, India) implant was placed between 26 and 27 (Fig. 6). The patient was recalled after 1 week to check the stability of the implant. When the implant was stable, a force of 100 to $150 \mathrm{gm}$ was applied with an E-chain. The force was measured with a Dontrix force gauge. The patient was recalled every 2 weeks to evaluate the intrusion. A 3.5-mm intrusion was achieved within 3 months with an additional 1-month retention period, to prevent relapse until crown placement in 36.

\section{Final Prosthesis}

Once the minimum space required for a cement-retained implant crown was achieved, a close-tray impression was made using a single-stage addition of silicone impression material. A metal-ceramic crown was fabricated and cemented. Postcementation clinical and radiographic follow-ups were done up to 6 months (Fig. 7).

\section{Case 2}

A 35-year-old male patient reported with a chief complaint of missing lower right second premolar and first molar $(45,46)$ and desired for replacement of the same. The teeth were extracted 1 year earlier due to extensive caries. On clinical examination, the patient presented with edge-to-edge bite and a class I molar relationship on the left side and mutilated occlusion on the right side. Upper right first molar (16) was supra-erupted reducing the

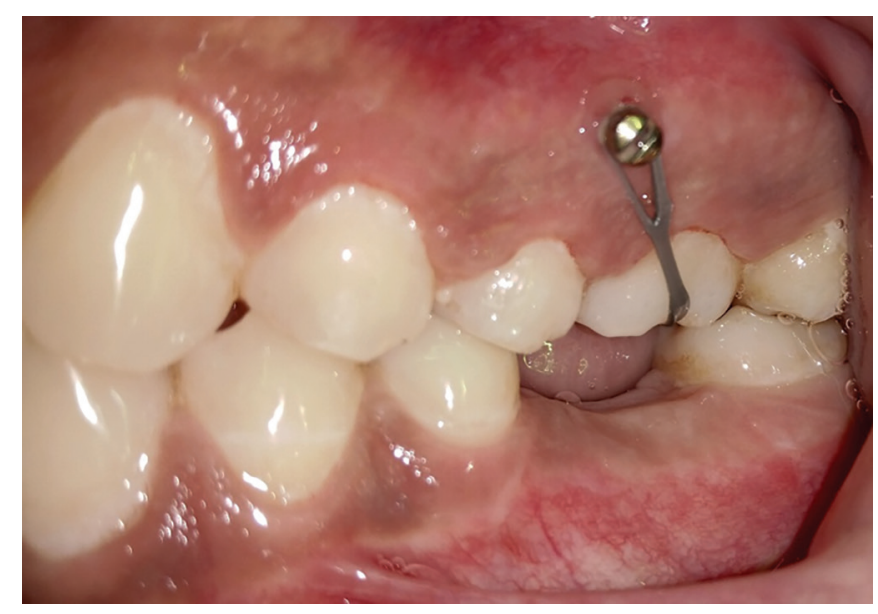

Fig. 6: Orthodontic mini-implant with E-chain with evident intrusion of 26

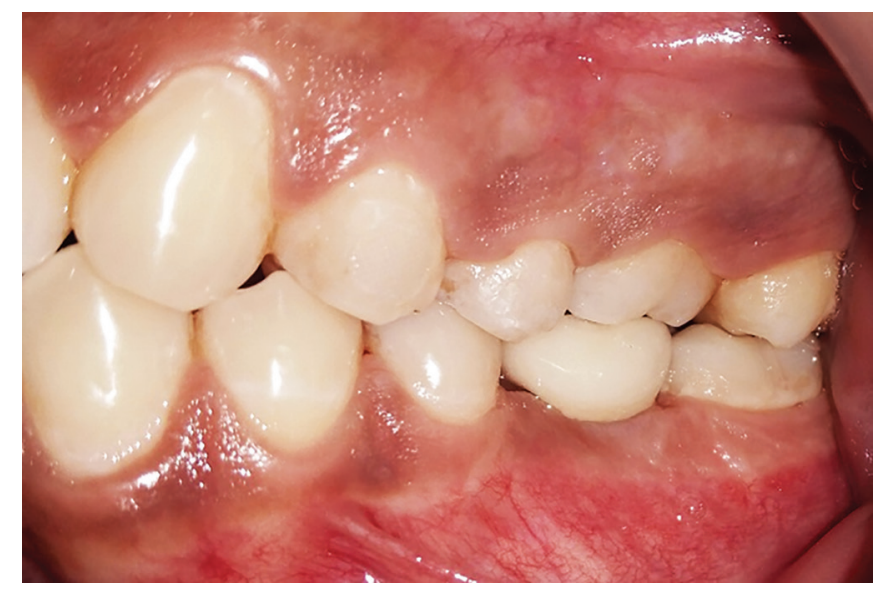

Fig. 7: Six-month postcrown placement with ideal intercuspation and class I molar relationship

space between the mandibular ridge crest and buccal cusp of 16 to $4 \mathrm{~mm}$ (Fig. 8). For the replacement of 46, either dental implant or fixed partial denture prosthesis was suggested. The patient opted for an implant prosthesis.

Similar to the first case, diagnostic casts were prepared and mounted. The treatment plan included placement of two implants in 45, 46 region with simultaneous intrusion of 16 using mini-implants.

\section{Implant Placement in the Mandible}

Similar to the previous case, radiographic examination and bone mapping were done followed by surgical stent preparation. A conventional mucoperiosteal flap was reflected and two implants, $4.2 \times 11.5 \mathrm{~mm}$ in 45 region and $5 \times 10 \mathrm{~mm}$ in 46 (Noris Medical, Israel), were placed and the flap was sutured. The implant was allowed to osseointegrate for 3 months.

\section{Mini-implant Placement in Maxilla}

One month prior to dental implant placement in the mandible, orthodontic mini-implants of the same diameter 

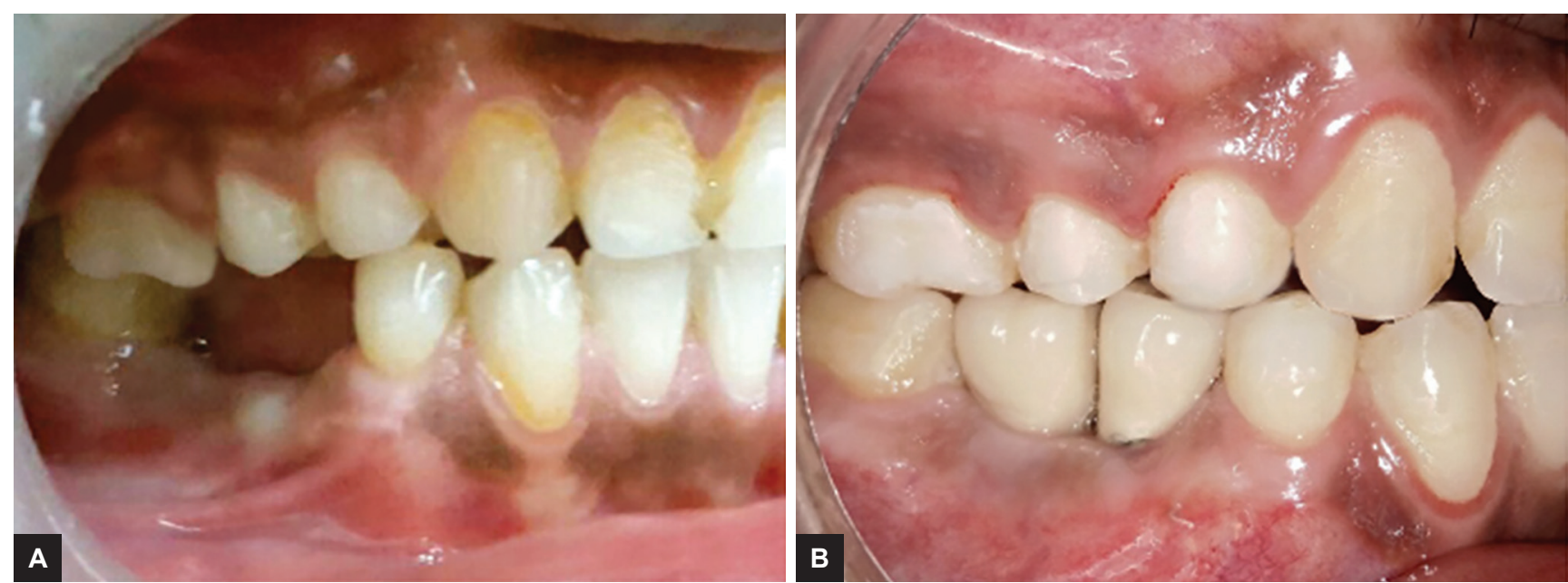

Figs $8 \mathrm{~A}$ and B: Pretreatment and posttreatment clinical photograph of case 2

and length were placed in the maxillary arch on the buccal side between 15 and 16 and on the palatal side between 16 and 17. The same recall protocol was followed as for the previous case. Intrusion of $2.5 \mathrm{~mm}$ was achieved in 3 months and a retention period of 1 month was provided, until crown placement.

\section{Final Prosthesis}

Once the minimum space required for a cement-retained implant crown was achieved, impressions were made and metal-ceramic crowns were fabricated and cemented (Fig. 8).

\section{DISCUSSION}

The CHS for implant dentistry is measured from the crest of the bone to the plane of occlusion. The minimum $\mathrm{CHS}$ needed for a fixed implant prosthesis should be $8 \mathrm{~mm}{ }^{4}$

Critical evaluation of the available restorative space during the diagnostic phase of implant treatment is necessary to determine the available prosthetic alternatives. ${ }^{5}$ Attempt to fabricate a prosthesis with inadequate restorative space may result in physiologically inappropriate counter, structurally weak prosthesis and esthetic compromise, and/or suboptimal retention and stability of the treated results. ${ }^{6}$

Loss of teeth in the posterior region results in extrusion of antagonist teeth, posing a problem during prosthetic rehabilitation. Hence, correction of this frequently encountered problem is deemed necessary prior to implant prosthesis planning either by conventional gross tooth reduction following endodontic therapy or by orthodontic intrusion with TADs for gaining the lost vertical space.

Anchorage is the major concern during orthodontic tooth movement, especially in cases where supra-eruption of upper molar occurs into the extraction site. Treating such minor discrepancies with the aid of conventional mechanotherapy may lead to anchor loss. With the help of TADs, it is possible to intrude a molar by applying a force from the buccal and palatal sides engaging an E-chain with minimal to no anchor loss. ${ }^{7}$ In the present cases, mini-implants were placed at the level of center of resistance of tooth on both sides, which resulted in bodily intrusive movement of upper molar.

Orthodontic mini-implants are simple and excellent anchorage devices, demanding less cooperation from patients and provide an opportunity for clinicians to perform desired tooth movements without unnecessarily sacrificing healthy tooth structure of antagonist tooth. ${ }^{8}$ In the present cases, ideal intercuspation was achieved at the treated sites in centric occlusion. A 6-month followup showed stable treatment results with a good level of patient satisfaction.

\section{CONCLUSION}

The present twin case report demonstrates the successful role of mini-implants in molar intrusion for establishing an ideal CHS for implant prosthesis to reestablish masticatory function.

\section{CLINICAL SIGNIFICANCE}

The twin case report demonstrates mini-implantsupported orthodontic molar intrusion as a convenient and minimally invasive replacement for conventional gross tooth reduction, for regaining the vertical space in cases of supra-eruption of maxillary molar associated with edentulism.

\section{REFERENCES}

1. Cope JB. Temporary anchorage devices in orthodontics: a paradigm shift. Semin Orthod 2005 Mar;11(1):3-9. 
2. Profitt WR, Fields HW, Sarver DM. Contemporary orthodontics. 5th ed. St. Louis, MO: Elsevier Publishers; 2013.

3. Misch CE, Wanh HL, Misch CM, Sharawy M, Lemons J, Judy KW. Rationale for application of immediate load in implant dentistry: Part I. Implant Dent 2004 Sep;13(3):207-215.

4. Misch CE. Contemporary implant dentistry. 3rd ed. St. Louis, MO: Elsevier Publishers; 2013.

5. Abujamra NF, Stavridakis MM, Miller RB. Evaluation of interarch space foe implant restorations in edentulous patients. A laboratory technique. J Prosthodont 2000 Jun;9(2): 102-105.
6. Chaimattayompol N, Arbree NS. Assessing the space limitation inside a complete denture for implant attachments. J Prosthet Dent 2003 Jan;89(1):82-85.

7. Papadopoulos MA, Tarawneh F. The use of miniscrew implants for temporary skeletal anchorage in orthodontics: a comprehensive review. Oral Surg Oral Med Oral Pathol Oral Radiol Endod 2007 May;103(5):e6-e15.

8. Shinde PH, Jain NV, Duggal R. Maxillary molar intrusion using Mini-implants to gain vertical space in the antagonist arch for rehabilitation with implant prosthesis: an interdisciplinary approach. Int J Clin Implant Dent 2015 Sep-Oct;1(3):119-122. 